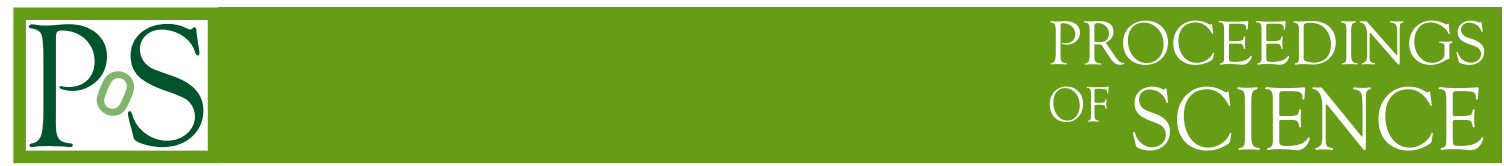

\title{
Radiative B decays and new physics searches at BABAR
}

\section{Liang SUN ${ }^{* \dagger}$}

University of Cincinnati

E-mail: sunleslac.stanford.edu

In this talk, a number of recent results on radiative $B$ decays from $B A B A R$ are covered. Based on the full $B A B A R$ dataset of 471 million $\Upsilon(4 S) \rightarrow B \bar{B}$ events, the inclusive decays $B \rightarrow X_{s} \gamma$ and $B \rightarrow X_{s} \ell^{+} \ell^{-}$, as well as the exclusive decays $B \rightarrow K \pi^{+} \pi^{-} \gamma$ are measured for new physics searches.

XXII. International Workshop on Deep-Inelastic Scattering and Related Subjects 28 April - 2 May 2014

Warsaw, Poland

*Speaker.

${ }^{\dagger}$ Special thanks to the conference organizers. 


\section{Introduction}

Both the $b \rightarrow s \gamma$ and $b \rightarrow s \ell^{+} \ell^{-}$transitions are flavor-changing neutral-current processes, and forbidden at tree level in the Standard Model (SM). The Feymann diagrams representing these types of transitions must involve loops. In the effective field theory for $b \rightarrow s$ transitions, the effective Hamiltonian can be written conventionally as $H_{E f f} \propto \sum_{i=1}^{10} C_{i} \mathcal{O}_{i}$, which factorizes shortdistance physics represented by the Wilson coefficients $C_{i}$ from long-distance effects. New physics beyond the SM brings in new loops for $b \rightarrow s$ transitions, and may change the SM values of Wilson coefficients.

During its entire data-taking period from 1999 till 2008, the $B A B A R$ detector [1] collected $\sim 471$ million $B \bar{B}$ pairs at the $\Upsilon(4 S)$ resonance with the $P E P-I I$ asymmetric energy $e^{+} e^{-}$collider. Based on the full $B A B A R$ dataset, we present here the search for direct $C P$ violation in $B \rightarrow X_{s} \gamma$ using a sum of exclusive final states, as well as the time-dependent analysis of $B^{0} \rightarrow K_{S}^{0} \pi^{+} \pi^{-} \gamma$ and the study of the $K^{+} \pi^{-} \pi^{+}$system in the decay $B^{+} \rightarrow K^{+} \pi^{-} \pi^{+} \gamma^{1}$. Finally we present the measurement of the $B \rightarrow X_{s} \ell^{+} \ell^{-}$branching fraction (BF) and search for direct $C P$ violation using a sum of exclusive final states. Here $X_{S}$ represents any hadronic system with one unit of strangeness.

For all the $B A B A R$ analyses presented here, the major backgrounds are either from $e^{+} e^{-} \rightarrow q \bar{q}$ ( $q=u, d, s, c)$ continuum events or from combinatorial $B \bar{B}$ events. Different types of multivariate classifiers, such as a Fisher discriminant, boosted decision trees (BDTs), and random forests, are trained for the background suppression. Furthermore, We distinguish a fully reconstructed $B$ candidate from backgrounds with energy-substituted mass $m_{\mathrm{ES}}=\sqrt{E_{\mathrm{CM}}^{2} / 4-p_{B}^{* 2}}$, and the energy difference $\Delta E=E_{B}^{*}-E_{\mathrm{CM}} / 2$, where $p_{B}^{*}$ and $E_{B}^{*}$ are the reconstructed $B$ momentum and energy in the $\Upsilon(4 S)$ center-of-mass $(\mathrm{CM})$ frame, respectively, and $E_{\mathrm{CM}}$ is the total CM energy.

\section{Search for direct $C P$ violation in $B \rightarrow X_{s} \gamma$ using a sum of exclusive final states}

The direct $C P$ asymmetry $\left(A_{C P}\right)$ for the sum of exclusive final states of $B \rightarrow X_{s} \gamma$ is measured by:

$$
A_{C P}\left(B \rightarrow X_{s} \gamma\right) \equiv \frac{\Gamma_{\bar{B}^{0} / B^{-} \rightarrow X_{s} \gamma}-\Gamma_{B^{0} / B^{+} \rightarrow X_{\bar{s}} \gamma}}{\Gamma_{\bar{B}^{0} / B^{-} \rightarrow X_{s} \gamma}+\Gamma_{B^{0} / B^{+} \rightarrow X_{\bar{s}} \gamma}}
$$

In the $\mathrm{SM}, A_{C P}$ is expected to be small and within a range of $-0.6 \%<A_{C P}^{S M}<2.8 \%$ [2]. Measuring the difference in $A_{C P}$ in charged and neutral $B$ mesons $\Delta A_{X_{s} \gamma} \equiv A_{B^{ \pm} \rightarrow X_{s} \gamma}-A_{B^{0} / \bar{B}^{0} \rightarrow X_{s} \gamma}$ is proposed to be another test of the SM. $\Delta A_{X_{s} \gamma}$ depends on two Wilson coefficients $C_{7 \gamma}$ and $C_{8 g}$ representing the electromagnetic dipole and the chromo-magnetic dipole transitions, respectively, according to the relationship [2],

$$
\Delta A_{X_{s} \gamma} \simeq 0.12 \times \frac{\tilde{\Lambda}_{78}}{100 \mathrm{MeV}} \operatorname{Im}\left(C_{8 g} / C_{7 \gamma}\right),
$$

where the interference amplitude $\tilde{\Lambda}_{78}$ is known to be within the range $17 \mathrm{MeV}<\tilde{\Lambda}_{78}<190 \mathrm{MeV}$. In the SM, we expect $\Delta A_{X_{s} \gamma}=0$ as the two Wilson coefficients $C_{8 g}$ and $C_{7 \gamma}$ are real. Experimentally, $C_{8 g}$ is not as well constrained as $C_{7 \gamma}$. Together with existing knowledge on $C_{7 \gamma}$, a measurement on $\Delta A_{X_{s} \gamma}$ can place a constraint on $C_{8 g}$.

\footnotetext{
${ }^{1}$ Charge conjugation is implied throughout unless explicitly noted.
} 

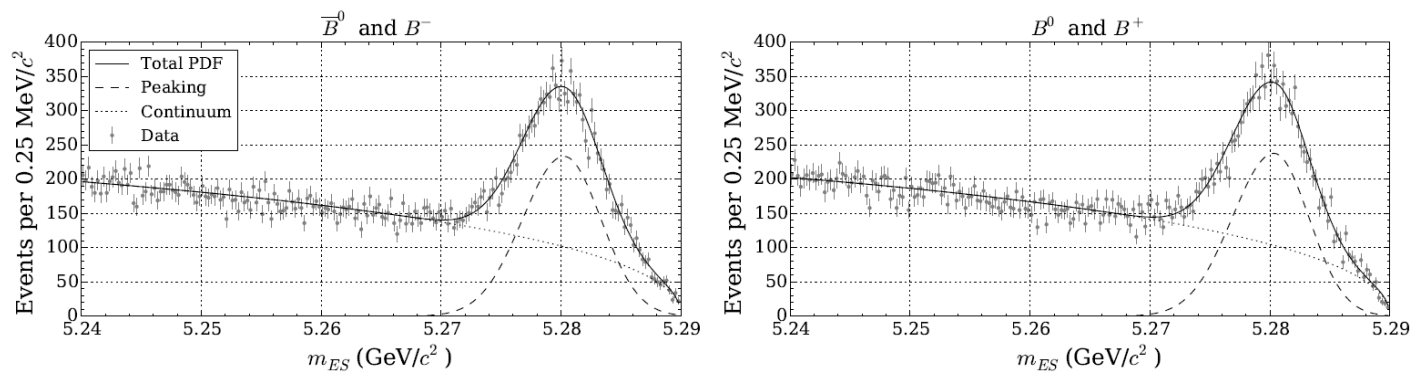

Figure 1: Fits to the $\bar{B}$ and $B$ samples.
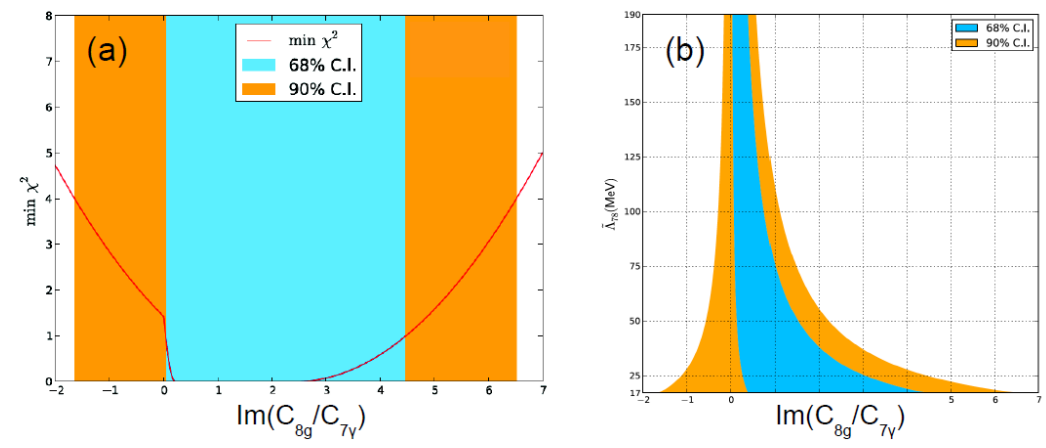

Figure 2: (a) The minimum $\chi^{2}$ for given $\operatorname{Im} \frac{C_{8 g}}{C_{7 \gamma}}$ from all possible values of $\tilde{\Lambda}_{78}$, also shown are the $68 \%$ and 90\% confidence intervals for $\operatorname{Im} \frac{C_{8 g}}{C_{7 \gamma}}$; (b) The $68 \%$ and $90 \%$ CLs for $\operatorname{Im} \frac{C_{8 g}}{C_{7 \gamma}}$ as a function of $\tilde{\Lambda}_{78}$.

In this analysis [3], we fully reconstruct $B$ meson decays in 16 self-tagging final states as listed in Ref. [3] where the $B$ flavor can be determined from the final state particles.

The raw asymmetry is extracted via fitting simultaneously to the $m_{\mathrm{ES}}$ distributions of $B$ and $\bar{B}$ tagged samples, as demonstrated in Fig. 1, which is further corrected for detector related effects to get $A_{C P}$. We measure $A_{C P}=+(1.7 \pm 1.9$ [stat.] \pm 1.0 [syst.])\%, which is the most precise to date and agrees with the SM. Through fitting simultaneously to the separate charged and neutral $B$ samples, we also provide the first measurement on $\Delta A_{X_{s} \gamma}: \Delta A_{X_{s} \gamma}=+(5.0 \pm 3.9$ [stat.] \pm 1.5 [syst.])\%, which is consistent with the SM expectation of zero. We compare the measured $\Delta A_{X_{s} \gamma}$ with the prediction based on the relationship in Eq. 2.2 for given $\operatorname{Im}\left(C_{8 g} / C_{7 \gamma}\right)$ and $\tilde{\Lambda}_{78}$ to calculate the minimum $\chi^{2}$, as shown in Fig. 2(a). The ranges of $\operatorname{Im}\left(C_{8 g} / C_{7 \gamma}\right)$ and $\tilde{\Lambda}_{78}$ that yield the minimum $\chi^{2}$ less than 1 and 4 are used to obtain the 68\% and 90\% confidence limits (CLs) shown in Fig. 2(b). Using the extremes in Fig. 2(b) for all permitted values of $\tilde{\Lambda}_{78}$, we conservatively $\operatorname{set} \operatorname{Im}\left(C_{8 g} / C_{7 \gamma}\right)$ : $0.07 \leq \operatorname{Im}\left(C_{8 g} / C_{7 \gamma}\right) \leq 4.48 @ 68 \% \mathrm{CL} ;-1.64 \leq \operatorname{Im}\left(C_{8 g} / C_{7 \gamma}\right) \leq 6.52 @ 90 \% \mathrm{CL}$.

\section{Studies of $B \rightarrow K \pi \pi \gamma$ decays}

We measure $C P$ asymmetry in the decay $B^{0} \rightarrow K_{S}^{0} \rho^{0} \gamma$ as a function of $B^{0}-\bar{B}^{0}$ decay-time difference $\Delta t$, which is defined as:

$$
A_{C P} \equiv \frac{\Gamma\left(\bar{B}^{0}(\Delta t) \rightarrow f_{C P} \gamma\right)-\Gamma\left(B^{0}(\Delta t) \rightarrow f_{C P} \gamma\right)}{\Gamma\left(\bar{B}^{0}(\Delta t) \rightarrow f_{C P} \gamma\right)+\Gamma\left(B^{0}(\Delta t) \rightarrow f_{C P} \gamma\right)}=\mathcal{S}_{K_{S}^{0} \rho^{0} \gamma} \sin \left(\Delta m_{d} \Delta t\right)-\mathcal{C}_{f_{K_{S}^{0}}^{0} \rho_{\gamma}} \cos \left(\Delta m_{d} \Delta t\right)
$$



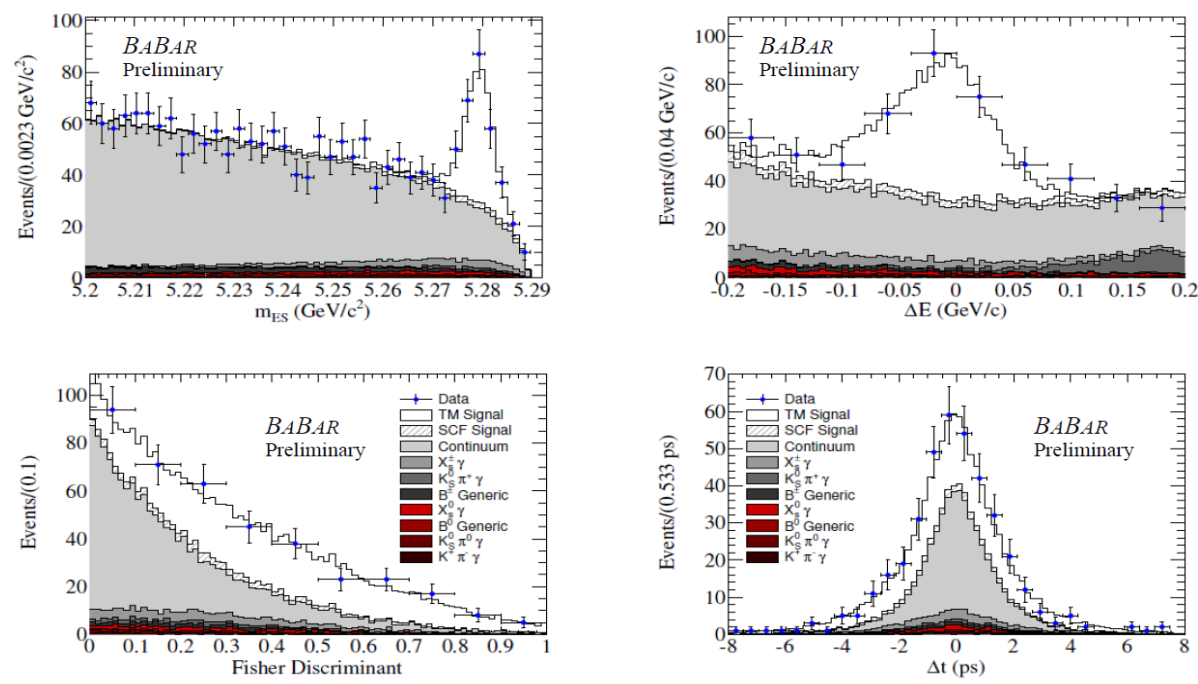

Figure 3: Fit to the distributions of $m_{\mathrm{ES}}$ (top left), $\Delta E$ (top right), and the Fisher-discriminant output (bottom left), and $\Delta t$ (bottom right) from the $B^{0} \rightarrow K_{S}^{0} \pi^{+} \pi^{-} \gamma$ data sample. The distributions have their signal/background ratios enhanced by means of the following requirements respectively: $-0.15<\Delta E<$ $0.10 \mathrm{GeV}\left(m_{\mathrm{ES}}\right) ; m_{\mathrm{ES}}>5.27 \mathrm{GeV} / c^{2}(\Delta E) ; m_{\mathrm{ES}}>5.27 \mathrm{GeV} / c^{2}$ and $-0.15<\Delta E<0.10 \mathrm{GeV}$ (Fisher and $\Delta t)$.

where $\Delta m_{d}$ is the $B^{0} \bar{B}^{0}$ oscillation frequency fixed to the measurement in Ref. [4], while $\mathcal{C}_{K_{S}^{0} \rho^{0} \gamma}$ and $\mathcal{S}_{K_{S}^{0} \rho^{0} \gamma}$ are the direct and mixing-induced $C P$ asymmetry parameters, respectively.

Experimentally, for the hadronic part of $B^{0} \rightarrow K_{S}^{0} \pi^{+} \pi^{-} \gamma$ final state, as $\rho^{0}(770)$ possesses a large natural width, a large amount of irreducible background events from non- $C P$ eigenstates $\left(K^{* \pm} \pi^{\mp}\right)$ will lie underneath the $\rho^{0}(770)$ resonance from our $B \rightarrow K_{S}^{0} \rho^{0}\left(\rightarrow \pi^{+} \pi^{-}\right) \gamma$ signal decay, and thus dilute our $C P$ parameter $\mathcal{S}_{K_{S}^{0} \rho^{0} \gamma}$. The dilution factor is defined as $\mathcal{D}_{K_{S}^{0} \rho^{0} \gamma} \equiv \frac{\mathcal{S}_{K_{S}^{0} \pi^{+} \pi^{-} \gamma}}{\mathcal{S}_{K_{S}^{0} \rho^{0} \gamma}}$, where $\mathcal{S}_{K_{S}^{0} \pi^{+} \pi^{-} \gamma}$ is the effective value of the mixing-induced $C P$ asymmetry measured based on all $B^{0} \rightarrow K_{S}^{0} \pi^{+} \pi^{-} \gamma$ data events. In order to measure $\mathcal{S}_{K_{S}^{0} \rho^{0} \gamma}$, an amplitude study on the hadronic system of $B^{0} \rightarrow K_{S}^{0} \pi^{+} \pi^{-} \gamma$ events is needed here to extract $\mathcal{D}_{K_{S}^{0} \rho^{0} \gamma}$. However this turns out to be a rather difficult task due to limited statistics. We therefore turn to the statistically more abundant sample of charged decays $B^{+} \rightarrow K^{+} \pi^{-} \pi^{+} \gamma$ for the determination of the dilution factor under the assumption of isospin symmetry.

The fits to $B^{+} \rightarrow K^{+} \pi^{-} \pi^{+} \gamma$ events undergo in three steps: first we perform a three-dimensional fit using $m_{\mathrm{ES}}, \Delta E$, and the Fisher discriminant output to unfold the signal distributions of invariant masses $m_{K \pi \pi}$ and $m_{K \pi}$. Then we fit to the $m_{K \pi \pi}$ distribution from the first step to determine the BFs of different kaonic resonances, such as $K_{1}(1270)^{+}, K_{2}^{*}(1430)^{+}$, etc. The final fit is to the $m_{K \pi}$ distribution to determine the amplitudes and BFs of the intermediate resonances $\left(K^{* 0}(892), \rho(770)^{0}\right.$, etc.) decaying to $K^{+} \pi^{-}$and $\pi^{+} \pi^{-}$. From the measured branching fractions in the last step of fit we compute the dilution factor as $\mathcal{D}_{K_{S}^{0} \rho^{0} \gamma}=0.549_{-0.094}^{+0.096}$, where the uncertainties incorporate both statistical and systematic contributions.

As shown in Fig. 3, we perform a four-dimensional fit to the data from the neutral mode $B^{0} \rightarrow$ $K_{S}^{0} \pi^{+} \pi^{-} \gamma$, to extract the $C P$ asymmetry parameters. With the measured dilution factor from the 

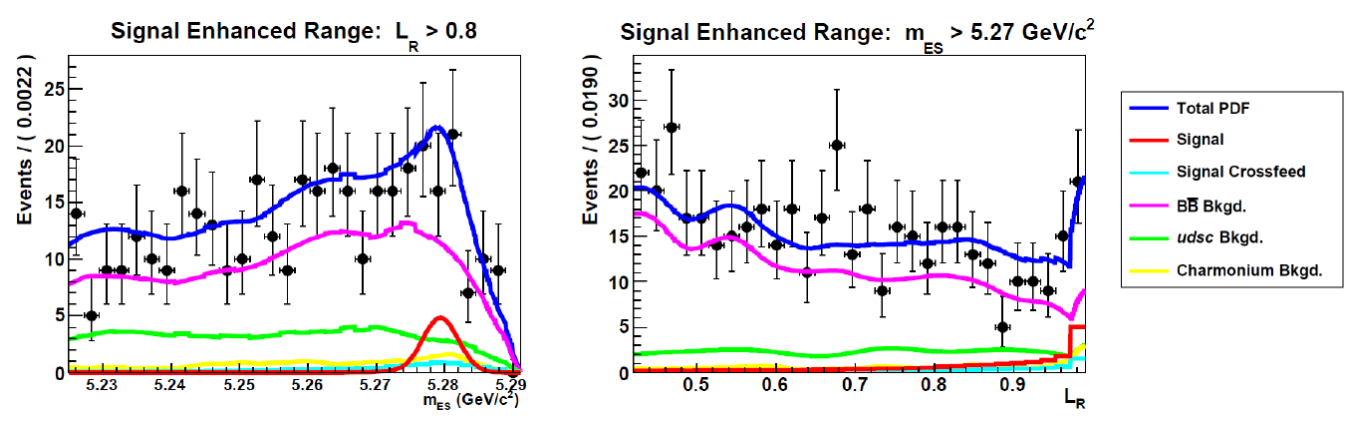

Figure 4: Fits to the distributions of $m_{\mathrm{ES}}$ (left) and $L_{R}$ (right) for $B \rightarrow X_{s} e^{+} e^{-}$events in the range $2.0<q^{2}<$ $4.3 \mathrm{GeV}^{2} / c^{4}$.

charged mode, we find for the $B^{0} \rightarrow K_{S}^{0} \rho^{0} \gamma$ decay $\mathcal{S}_{K_{S}^{0} \rho^{0} \gamma}=\frac{\mathcal{S}_{K_{S}^{0} \pi^{+} \pi^{-} \gamma}}{\mathcal{D}_{K_{S}^{0} \rho^{0} \gamma}}=0.249 \pm 0.455$ [stat. $]_{-0.060}^{+0.076}[$ syst.], which is compatible with the SM expectation of $\sim 0.03[5]$.

\section{Branching fractions and direct $C P$ asymmetry in $B \rightarrow X_{s} \ell^{+} \ell^{-}$using a sum of exclusive final states}

In this analysis[6], the inclusive decay $B \rightarrow X_{S} \ell^{+} \ell^{-}$is studied in 20 exclusive final states each comprises one kaon and at most two pions, $\ell^{+} \ell^{-}$is either $e^{+} e^{-}$or $\mu^{+} \mu^{-}$, as listed in Ref. [6]. We reject events with dilepton mass squared $q^{2} \equiv m_{\ell \ell}^{2}$ within a range of $6.8<q^{2}<10.1 \mathrm{GeV}^{2} / c^{4}$ $\left(12.9<q^{2}<14.2 \mathrm{GeV}^{2} / c^{4}\right)$ to suppress signal-like charmonium backgrounds with $J / \psi(\psi(2 S))$ from $B$ decays. The entire event selection criteria represent $\sim 70 \%$ of the inclusive $B \rightarrow X_{s} \ell^{+} \ell^{-}$ rate with the invariant mass of the hadronic system $m_{X_{s}}<1.8 \mathrm{GeV} / c^{2}$, accounting for $K_{L}^{0}$ modes, $K_{S}^{0} \rightarrow \pi^{0} \pi^{0}$, and $\pi^{0}$ Dalitz decays in our signal reconstruction efficiencies. We also extrapolate for the missing final states, and those with $m_{X_{s}}>1.8 \mathrm{GeV} / c^{2}$, using JETSET fragmentation [7] and theory predictions.

We measure the total $\mathrm{BF}$ and partial BFs in different ranges of $q^{2}$ or $m_{X_{s}}$ by performing a two-dimensional fit to the distributions of $m_{\mathrm{ES}}$ and likelihood ratio $L_{R}$, which is defined as $L_{R} \equiv$ $\mathcal{P}_{S} /\left(\mathcal{P}_{S}+P_{B}\right)$ with $\mathcal{P}_{S}\left(\mathcal{P}_{B}\right)$ as the probability for a correctly-reconstructed signal ( $B \bar{B}$ background) event calculated based on the response of $B \bar{B}$ BDT. The $L_{R}$ distributions for signal and $B \bar{B}$ background events peak around one and zero, respectively. A fit example is shown in Fig. 4 for $B \rightarrow X_{s} e^{+} e^{-}$events with $2.0<q^{2}<4.3 \mathrm{GeV}^{2} / c^{4}$.

Our measured partial BF results are shown in Fig. 5. We find the total BF for $q^{2}>0.1 \mathrm{GeV}^{2} / c^{4}$ to be $\mathcal{B}\left(B \rightarrow X_{S} \ell^{+} \ell^{-}\right)=\left(6.73_{-0.63-0.25}^{+0.70+0.34} \pm 0.50\right) \times 10^{-6}$, which is less than $2 \sigma$ above the SM prediction of $\mathcal{B}_{\mathrm{SM}}\left(B \rightarrow X_{S} \ell^{+} \ell^{-}\right)=(4.6 \pm 0.8) \times 10^{-6}$ [8]. In the low mass range with $1<q^{2}<6 \mathrm{GeV}^{2} / c^{4}$, we have $\mathcal{B}^{\text {low }}\left(B \rightarrow X_{s} \ell^{+} \ell^{-}\right)=\left(1.60_{-0.39-0.13}^{+0.41+0.17} \pm 0.18\right) \times 10^{-6}$, which is in good agreement with the SM predictions of $\mathcal{B}_{\mathrm{SM}}^{\text {low }}\left(B \rightarrow X_{s} e^{+} e^{-}\right)=(1.64 \pm 0.11) \times 10^{-6}$ and $\mathcal{B}_{\mathrm{SM}}^{\text {low }}\left(B \rightarrow X_{s} \mu^{+} \mu^{-}\right)=$ $(1.59 \pm 0.11) \times 10^{-6}$ [9]. And in the high mass range with $q^{2}>14.2 \mathrm{GeV}^{2} / c^{4}$, our result of $\mathcal{B}^{\text {high }}\left(B \rightarrow X_{S} \ell^{+} \ell^{-}\right)=\left(0.57_{-0.15-0.02}^{+0.16+0.03} \pm 0.00\right) \times 10^{-6}$ is about $2 \sigma$ higher than the SM predictions of $\mathcal{B}_{\mathrm{SM}}^{\text {high }}\left(B \rightarrow X_{s} e^{+} e^{-}\right)=(0.21 \pm 0.07) \times 10^{-6}$ and $\mathcal{B}_{\mathrm{SM}}^{\text {high }}\left(B \rightarrow X_{s} \mu^{+} \mu^{-}\right)=(0.24 \pm 0.07) \times 10^{-6}[9]$. In all the three results listed above, the first uncertainties are statistical, the second experimental systematics and the third model-dependent systematics. 

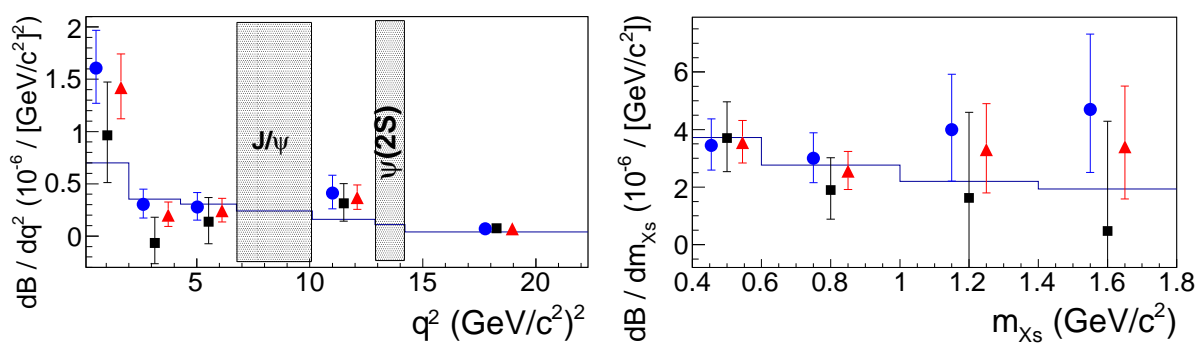

Figure 5: Differential BF as a function of $q^{2}$ (left) or $m_{X_{s}}$ (right) for $X_{s} e^{+} e^{-}(\bullet), X_{s} \mu^{+} \mu^{-}(\mathbf{\square})$, and leptonflavor-averaged final states $(\boldsymbol{\Lambda})$. The errors correspond to the total uncertainties. The histogram shows the SM expectation, which has uncertainties of approximately $10-30 \%$ in different $q^{2}$ or $m_{X_{s}}$ regions. The shaded boxes in the left plot denote the vetoed charmonium regions.

In 14 self-tagging final states as listed in Ref. [6], we search for the direct $C P$ asymmetry in $B \rightarrow X_{s} \ell^{+} \ell^{-}$which is defined in the same manner as $A_{C P}\left(B \rightarrow X_{s} \gamma\right)$ in Eq. 2.1. $A_{C P}\left(B \rightarrow X_{s} \ell^{+} \ell^{-}\right)$ is expected to be well below $1 \%$ in the SM [10]. We find the total $A_{C P}$ for $q^{2}>0.1 \mathrm{GeV}^{2} / c^{4}$ to be $A_{C P}\left(B \rightarrow X_{S} \ell^{+} \ell^{-}\right)=0.04 \pm 0.11$ [stat.] \pm 0.01 [syst.]. We also measure $A_{C P}$ in different $q^{2}$ regions, and find all the results to be consistent with zero as expected in the SM.

\section{Summary}

With the full $B A B A R$ dataset of 471 million $B \bar{B}$ pairs, we perform a series of interesting measurements in radiative $B$ decays recently. Our direct $A_{C P}\left(B \rightarrow X_{s} \gamma\right)$ result is the most precise result to date and agree with the SM. The measured mixing-induced $C P$ parameter for $B^{0} \rightarrow K_{S}^{0} \rho^{0} \gamma$ is found to be compatible with the SM expectation. Our $\mathcal{B}\left(B \rightarrow X_{s} \ell^{+} \ell^{-}\right)$and $\operatorname{direct} A_{C P}\left(B \rightarrow X_{s} \ell^{+} \ell^{-}\right)$ results are generally consistent with the SM predictions, however some tensions exist for the total $\mathrm{BF}$ and partial $\mathrm{BF}$ in the region of $q^{2}>14.2 \mathrm{GeV}^{2} / c^{4}$.

\section{References}

[1] B. Aubert et al. [BaBar Collaboration], Nucl. Instrum. Meth. A 479, 1 (2002); Nucl. Instrum. Meth. A 729, 615 (2013).

[2] M. Benzke, S. J. Lee, M. Neubert and G. Paz, Phys. Rev. Lett. 106, 141801 (2011).

[3] J. P. Lees et al. [BaBar Collaboration], arXiv:1406.0534 [hep-ex].

[4] B. Aubert et al. [BaBar Collaboration], Phys. Rev. Lett. 99, 171803 (2007).

[5] D. Atwood, T. Gershon, M. Hazumi, and A. Soni, Phys.Rev. D 71, 076003 (2005).

[6] J. P. Lees et al. [BaBar Collaboration], Phys. Rev. Lett. 112, 211802 (2014).

[7] T. Sjostrand, Computer Physics Commun. 82, 74 (1994).

[8] A. Ghinculov, T. Hurth, G. Isidori and Y. P. Yao, Nucl. Phys. B 685, 351 (2004).

[9] T. Huber, T. Hurth and E. Lunghi, Nucl. Phys. B 802, 40 (2008).

[10] D.S. Du and M.Z. Yang, Phys. Rev. D 54, 882 (1996); A. Ali and G. Hiller, Eur. Phys. J. C 8, 619 (1999); C. Bobeth, G. Hiller and G. Piranishvili, JHEP 0807, 106 (2008); W. Altmannshofer et al., JHEP 0901, 019 (2009). 